\title{
Systematic shell-model study on spectroscopic properties from light to heavy nuclei
}

\author{
Cenxi Yuan ${ }^{1, *}$ \\ ${ }^{1}$ Sino-French Institute of Nuclear Engineering and Technology, Sun Yat-Sen University, Zhuhai, \\ 519082, Guangdong, China
}

\begin{abstract}
A systematic shell-model study is performed to study the spectroscopic properties from light to heavy nuclei, such as binding energies, energy levels, electromagnetic properties, and $\beta$ decays. The importance of cross-shell excitation is shown in the spectroscopic properties of neutron-rich boron, carbon, nitrogen, and oxygen isotopes. A special case is presented for low-lying structure of ${ }^{14} \mathrm{C}$. The weakly bound effect of proton $1 s_{1 / 2}$ orbit is necessary for the description of the mirror energy difference in the nuclei around $\mathrm{A}=20$. Some possible isomers are predicted in the nuclei in the southeast region of ${ }^{132} \mathrm{Sn}$ based on a newly suggested Hamiltonian. A preliminary study on the nuclei around ${ }^{208} \mathrm{~Pb}$ are given to show the ability of the shell model in the heavy nuclei.
\end{abstract}

\section{Introduction}

Many neutron- and proton-rich nuclei are discovered and investigated through the new generation facilities. The nuclear shell model is one of the important methods to study the properties of nuclei from the stability line to the drip line, including the binding energies, energy levels, electromagnetic properties, $\beta$ decays, and many other properties $[1,2]$. But the required calculations are (much) beyond the present computational limitation for many nuclei. Some approximations are suggested to overcome the limitation, such as Monte-Carlo shell model [3], projected shell model [4], and so on. Although it is impossible to investigate all nuclei based on the shell-model calculation in a full model space, it is important to perform systematic shell-model study from light to heavy nuclei. A brief introduction of our recent shell-model works will be presented in the present paper.

\section{Shell-model Hamiltonians and results}

There are some shell-model Hamiltonians for psd model space, such as MK [5], WBT [6], WBP [6], and recently suggested YSOX [7]. YSOX is based on the monopole based universal interaction $V_{\mathrm{MU}}$ [8], SFO [9] and SDPF-M [10]. The binding energies, levels, electromagnetic properties, and $\mathrm{B}(\mathrm{GT})$ transitions of boron, carbon, nitrogen, and oxygen isotopes are well described by YSOX, especially the neutron drip line of carbon and oxygen isotopes. YSOX reconsidered the off-diagonal cross-shell interaction, which connects the $N \hbar \omega$ and

\footnotetext{
*e-mail: yuancx@mail.sysu.edu.cn
} 
$(N+2) \hbar \omega$ configurations, which should play an important role in certain states. For example, both the inclusion of $4 \hbar \omega$ configuration and the proper strength of the off-diagonal cross-shell interaction are necessary to describe the low-lying levels, electromagnetic and $\mathrm{B}(\mathrm{GT})$ transitions of ${ }^{14} \mathrm{C}$ [11]. The recently-observed negative states in ${ }^{17,19} \mathrm{C}$ are better described by YSOX comparing with the previous Hamiltonians [12].

The USD family is one of the most successful Hamiltonians in the $s d$ model space, including USD [13], USDA and USDB [14]. But USD family does not include the isospin asymmetry term and can not discribe mirror energy differences (MED) between the mirror partners. In the proton-rich nuclei around $A=20$, some MED are very large, which comes from the weakly bound proton $1 s_{1 / 2}$ orbit $[15,16]$. The USD family can well describe MED in the nuclei around $\mathrm{A}=20$ after modifications on single particle energies and two body matrix elements related to the proton $1 s_{1 / 2}$ orbit [16]. The modified Hamiltonian gives nice description on the recent measured mass, $\mathrm{B}(\mathrm{GT})$ strength and branch ratio of ${ }^{22} \mathrm{Si}$ [17].

The nuclei around ${ }^{132} \mathrm{Sn}$ attracts a lot of interests in the recent and coming experimental projects, such as recent works on ${ }^{140} \mathrm{Te}[18],{ }^{140} \mathrm{I}$ [19], and ${ }^{132} \mathrm{Cd}[20]$. A newly suggested Hamiltonian gives nice descriptions on neutron separation energies, levels, and lifetimes of some isomers in the south-east region of ${ }^{132} \mathrm{Sn}$ [21]. Some possible isomers are suggested in ${ }^{135} \mathrm{Sn},{ }^{132,133,134} \mathrm{In},{ }^{131} \mathrm{Cd},{ }^{130} \mathrm{Ag}$, and ${ }^{129,130} \mathrm{Pd}$ [21]. Just after the suggestion of the Hamiltonian, the first spectroscopic information in this region was published with the observation of a $618(8) \mathrm{keV} 21_{1}^{+}$state in ${ }^{132} \mathrm{Cd}[20]$, while the new Hamiltonian gives $708 \mathrm{keV}$.

The nuclear shell model provides nice descriptions in the nuclei around ${ }^{208} \mathrm{~Pb}$. But taking ${ }^{208} \mathrm{~Pb}$ as the core, the number of valence (hole) protons and neutrons can not be very large because of the computational limitation. Some further truncation on the occupancy in each orbit make the calculation on nuclei with more valence (hole) protons and neutrons possible. For example, the spin parity of the ground state of ${ }^{223} \mathrm{~Np}$ is observed through $\alpha$ decay and reproduced by the shell-model calculation in a truncated model space [22]. The original computational cost for ${ }^{223} \mathrm{~Np}$ is far beyond the present computational limitation. Some preliminary results on the binding energies and levels of lead, thallium, mercury, gold, and platinum were shown during CGS16 based on a Hamiltonian under preparation [23]. The root mean square between observed and shell-model results is only $158 \mathrm{keV}$ for the binding energies of 40 nuclei in these isotopes.

\section{Summary}

The shell model can calculate the medium and heavy mass nuclei with a core, such as doubly magic nuclei ${ }^{132} \mathrm{Sn}$ and ${ }^{208} \mathrm{~Pb}$. Some nuclei around ${ }^{132} \mathrm{Sn}$ and ${ }^{208} \mathrm{~Pb}$ are of highly importance and will be experimentally investigated in the near future. A systematic shell model study should provide many helpful spectroscopic information for the coming experimental study and feedbacks from the nuclear structure to the nuclear force. For example, the shellmodel spectroscopic properties for $\mathrm{N}=82$ and 132 isotones are expected to be important for $r$-process.

\section{Acknowledgment}

The present work has been supported by the National Natural Science Foundation of China (11775316), the Tip-top Scientific and Technical Innovative Youth Talents of Guangdong special support program (2016TQ03N575), the Pearl River S\&T Nova Program of Guangzhou (201506010060), the Special Program for Applied Research on Super Computation of the NSFC Guangdong Joint Fund (the second phase) (U1501501), and the Fundamental Research Funds for the Central Universities (17lgzd34). 


\section{References}

[1] B. A. Brown, Prog. Part. Nucl. Phys. 47, 517 (2001).

[2] E. Caurier, et al., Rev. Mod. Phys. 77, 427 (2005).

[3] T. Otsuka, et al., Prog. Part. Nucl. Phys. 47, 319 (2001).

[4] K. Hara and Y. Sun, Int. J. Mod. Phys. E 04, 637 (1995).

[5] D. J. Millener and D. Kurath, Nucl. Phys. A 255, 315 (1975).

[6] E. K. Warburton and B. A. Brown, Phys Rev. C 46, 923 (1992).

[7] C.X. Yuan, et al., Phys Rev. C 85, 064324 (2012).

[8] T. Otsuka, et al., Phys. Rev. Lett. 104, 012501 (2010).

[9] T. Suzuki, et al., Phys Rev. C 67, 044302 (2003).

[10] Y. Utsuno, et al., Phys Rev. C 60, 054315 (1999).

[11] C.X. Yuan, Chin. Phys. C, 41(10), 104102, (2017)

[12] J.W. Hwang, et al., Phys. Lett. B 769, 503 (2017).

[13] B. A. Brown and B. H. Wildenthal, Annu. Rev. Nucl. Part. Sci. 38, 29 (1988).

[14] B. A. Brown and W. A. Richter, Phys. Rev. C 74, 034315 (2006).

[15] K. Ogawa, et al., Phys. Lett. B 464, 157 (1999).

[16] C.X. Yuan, et al., Phys Rev. C 89, 044327 (2014).

[17] X.X. Xu, et al., Phys. Lett. B 766, 312 (2017); X.X. Xu, et al., arXiv:1610.08291.

[18] B. Moon, et al., Phys Rev. C 95, 044322 (2017).

[19] B. Moon, et al., Phys Rev. C 96, 014325 (2017).

[20] H. Wang, Phys. Rev. C 94, 051301(R) (2016).

[21] C.X. Yuan, et al., Phys. Lett. B 762, 237 (2016).

[22] M.D. Sun, et al., Phys. Lett. B 771, 303 (2017).

[23] C.X. Yuan, et al., in preparation. 